\title{
Study on wind turbine blade defect detection system based on imaging array
}

\author{
Ningning Zhang ${ }^{1}$, and Chengzhi Lu$^{1}$, Anmin Wang ${ }^{1}$ \\ ${ }^{1}$ Huadian Electric Power Research Institute Co., Ltd., Hangzhou, China
}

\begin{abstract}
Currently in the process of wind farm inspection, wind turbine blade appearance inspection mainly adopts the telescope or high-definition cameras, low detection efficiency, labor intensity and the precision is limited, in order to solve this problem, a kind of wind turbine blades defect recognition system based on image array is proposed. Through the joint of array camera and image processing server, the functions of the image acquisition, processing, and defect recognition and detection results output are implemented. The software of artificial intelligence deep learning based on neural network algorithm is used to identify the defects of blade image, and output quality analysis report, to realize automatic detection of wind turbine blade surface defect. The field measurement results show that the system greatly improves the efficiency and accuracy of wind turbine blade defect detection.
\end{abstract}

\section{The introduction}

Driven by China's policy of vigorously developing wind power, the whole wind power industry in China has been developing rapidly in recent years. By the end of 2018, China's total installed wind power capacity reached 184 million $\mathrm{kW}$, accounting for $9.7 \%$ of the total installed power capacity. With the rapid growth of wind power installation in China, wind turbines are developing towards large scale, high efficiency and intelligence. At the same time, the blade size is also increasing. Due to the complex and harsh working environment of wind turbines and the long-term alternating load, the blades are prone to crack, paint peeling, wear and other surface defects [1]. If the early damage is not found in time, it may develop into mechanical failure under bad working conditions. Once the accident happens, the consequences will be serious. Therefore, it is very necessary to conduct health monitoring and damage identification research on the leaves.

At present, the main nondestructive detection methods for wind turbine blades include vibration detection technology, acoustic emission detection technology, optical fiber sensing detection technology and infrared thermal imaging detection technology, and so on. Vibration detection technology can monitor the dynamic characteristic changes of blades by sticking vibration sensors on the surface of blades, so as to realize online fault diagnosis of wind turbine blades [2]. Luping $\mathrm{Li}$ et al. applied the vibration detection technology in the field of blade crack damage monitoring, and determined the crack damage location by studying the changes of vibration characteristics before and after the blade crack and at different damage locations and degrees [3]. Acoustic emission detection technology can determine the location and characteristics of blade damage through acoustic emission sensor reception and analysis. TANG et al. used AE(acoustic emission) to realize online monitoring of wind turbine blade structure health with a blade length of $45.7 \mathrm{~m}$, confirming that AE monitoring method can provide early warning for in-service wind turbine blade damage [4]. Fiber grating sensing detection technology is to diagnose the health status of wind turbine blades by monitoring the temperature, strain and other changes of wind turbine blades with fiber grating sensors. Due to its high sensitivity, small size and strong anti-interference ability, this detection method has been increasingly applied in state monitoring [5]. Lee $\mathrm{J} \mathrm{K}$ et al. used fiber Bragg grating sensor to realize strain monitoring of wind turbine blades health [6]. Because of the high testing cost, how to reduce the cost has become a key problem in the wind power industry. Infrared thermal imaging detection technology is to receive the infrared radiation of the blade through the infrared thermal imaging instrument, convert it into an image, and judge the damage of the blade according to the infrared imaging image. In recent years, more and more researchers at home and abroad have invested in extending infrared thermal imaging technology to online monitoring of in-service wind turbine blades damage. DOROSHTNASIR et al. by improving the infrared thermal image analysis method, can minimize the impact of external environmental interference and achieve the purpose of remote state monitoring of in-service wind turbine blades [7]. However, none of these technologies can identify the surface detail defects of wind turbine blades from multiple angles and provide real-time analysis.

In this paper, a wind turbine blade defect detection system is proposed with wind turbine blade images

Email :ningning-zhang@chder.com 
collected by camera array and image processing server. The image of the blade surface is collected by the camera array imaging equipment, which is transmitted to the image recognition system for image processing and defect extraction. Finally, the blade quality analysis report is output to achieve the purpose of rapid and efficient defect detection of the blade.

\section{Camera array and image processing system}

\subsection{Technical scheme of wind turbine blade defect detection system}

According to the field conditions of the wind farm, the camera array system for wind turbine blade image acquisition built in this paper is shown in Figure 1. The schematic diagram of the connection between camera array and image processing server is shown in Figure 2.

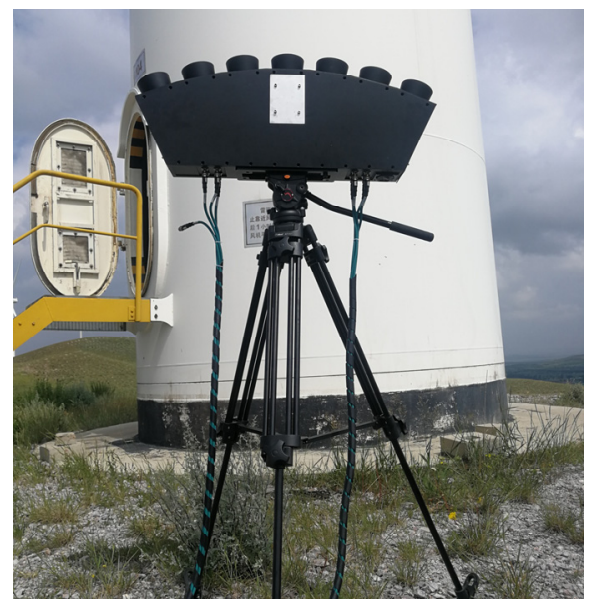

Fig. 1. Camera array system.

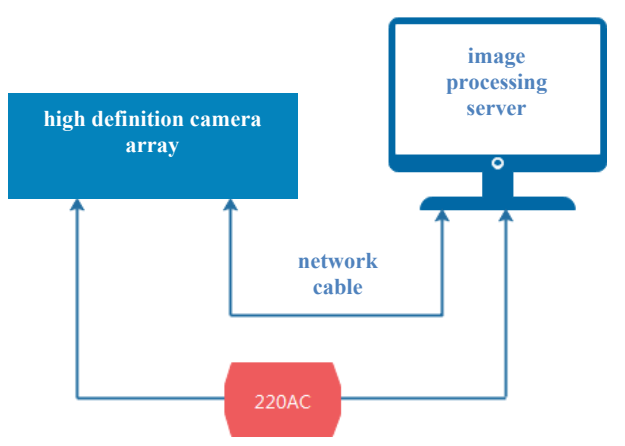

Fig. 2. Schematic diagram the connection between camera array and image processing server.

The connection relationship among camera array, wind turbine and image processing server is as follows:

- The whole set of equipment is physically divided into two parts: camera array and image processing server, which are connected by a communication cable for onsite assembly and disassembly.

- There are two sets of cameras in the camera array, one is a high-definition array, which is used to identify defects; The other is a positioning camera, which is used to determine whether the positioning blade fills the entire field of view and assist in adjusting the shooting angle of the camera array.

- The camera array is mounted on the cradle head to adjust the shooting angle.

- The camera array is connected to the computer by a network cable, with a length of up to $100 \mathrm{~m}$, which facilitates field wiring.

The working process of this system is shown in Figure 3:

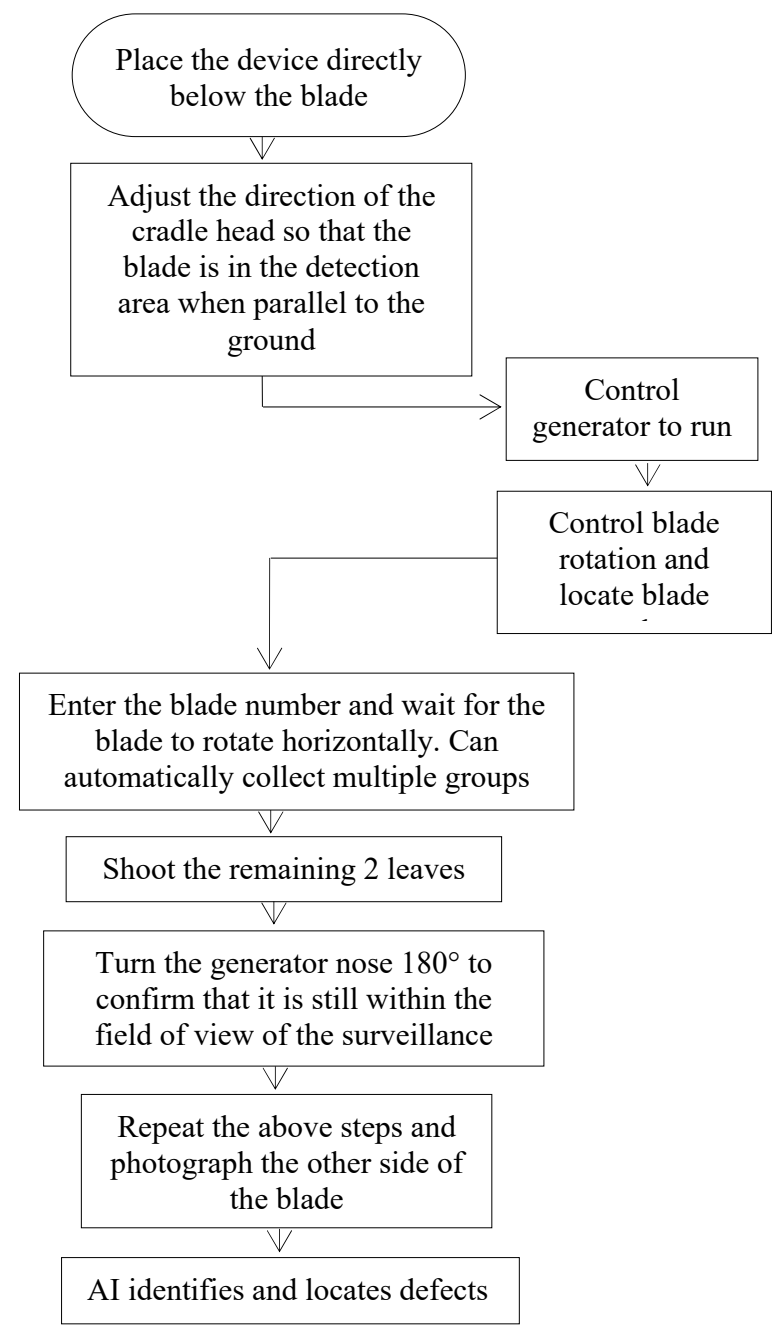

Fig. 3. Working process of the system

The system takes multi-angle photos of the stationary wind turbine blades by using a high-definition camera array mounted on the cradle head, and stores them in a computer. For one thing, the stored images are detected by the wind turbine blade defect detection system; for another thing, they are stored in the defect database and compared with the defect information from the system, so as to prepare for the accurate detection of blade defects and the output of blade quality analysis report.

\subsection{Artificial intelligence identification system}

After the camera array system completes the acquisition of wind turbine blade images, it is transmitted to the blade image processing system through communication 
cables. The collected blade images (supporting image formats: PNG, BMP, TIFF, JPEG) are calibrated and denoised, and the processed images are stored and analyzed.

This image recognition software (named: HD01) is an artificial intelligence deep learning software developed for machine vision based on neural network algorithm. It is mainly used for the location and recognition of target objects or defects, and also has the function of character recognition and picture classification. HD01 image recognition software can train by self-learning like human beings. Through big data learning and constant repetition, the accuracy rate of identifying defects is infinitely close to $100 \%$. The advantage of this software is that once the desired result cannot be detected in the field, and there is no need for software programming technicians to carry out software programming and debugging on the production site. The desired result can be achieved by learning the undetected picture on the production site again, with high detection accuracy and the minimum detected defect size of $5 \mathrm{~mm}$.

\subsubsection{Labeling and training of samples}

After the sample is imported, GT frame is labeled on the target that needs to be positioned and recognized, as shown in Figure 4.

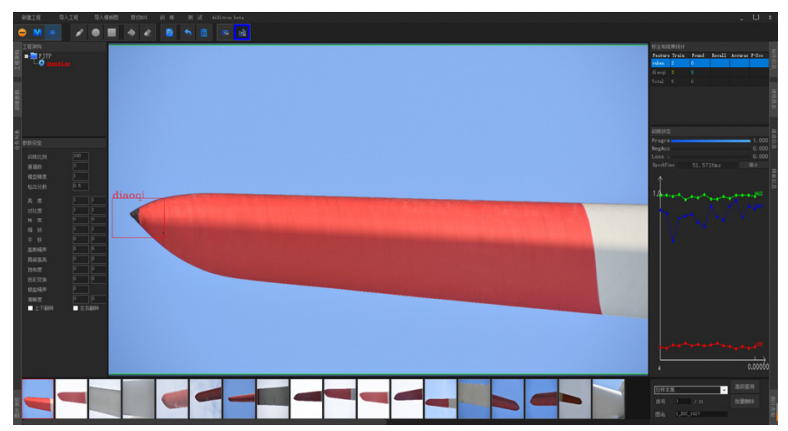

Fig. 4. Labeling of samples.

After all the samples are labeled, the samples can be "trained", as shown in Figure 5. After the training, the recognition effect of all samples will be automatically tested. The detection rate of defects depends on the number of training samples, and the larger the number of samples, the higher the detection rate.

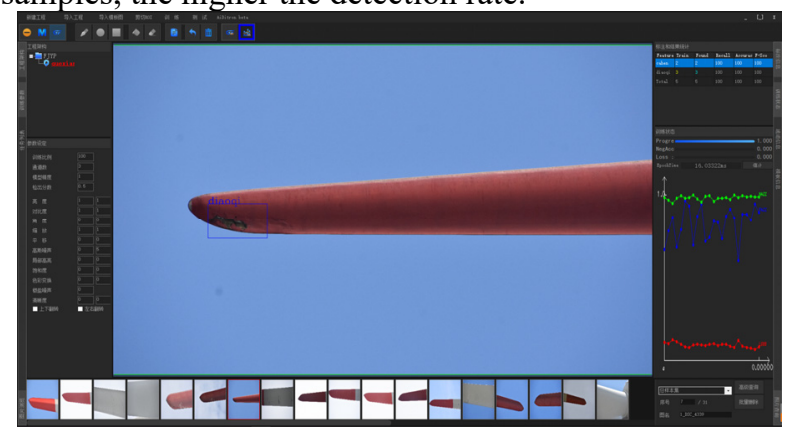

Fig. 5. Training of samples.

\subsubsection{Statistical description of system results}

The result statistics interface is shown in Figure 6.

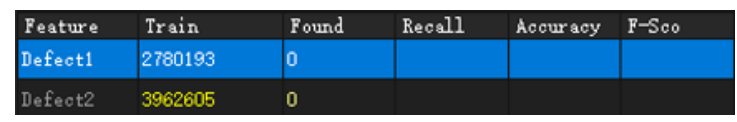

Fig. 6. Results statistical interface.

Feature: Categories of objectives;

Train: The number of targets to participate in the training, The unit of positioning module is the number of labeled rectangular boxes, the unit of pixel segmentation module is the number of labeled pixels, and the unit of classification module is the number of labeled pictures;

Found:The number of targets found at the end of the test;

Recall:Recall rate, number of correctly identified targets/number of targets participating in training (Train);

Precis: Recall accuracy, number of correctly identified targets/number of targets found (Found);

F-Sco:F-score $=(2 *$ precision $*$ recall $) /($ precision + recall $)$.

\section{Analysis of field test results}

In order to verify the feasibility of the wind turbine blade defect detection system established in this paper, researchers conducted a blade defect detection test in a wind farm in Taierzhuang, Shandong province in May 2019. The wind farm was connected to the grid in December 2015, with a single fan capacity of $2.0 \mathrm{MW}$, blade length of $54 \mathrm{~m}$, tower height of $80 \mathrm{~m}$, and a warranty period of 5 years, which is still in the warranty period. According to the feedback of wind farm personnel, two wind turbines with relatively more surface defects in the inspection process were selected for image collection in this test. The number of images collected by each one was about 200 , and the collection time was about 10 minutes. Finally, HD01 image recognition software was used to detect, classify and locate the collected images for defect types of surface cracks, paint peeling, surface corrosion and repair traces. The detection results are shown in Table 1.

Table 1. Detection results of leaf surface defect types.

\begin{tabular}{|c|c|c|c|c|}
\hline $\begin{array}{c}\text { Defect } \\
\text { types }\end{array}$ & $\begin{array}{c}\text { Identification } \\
\text { number }\end{array}$ & $\begin{array}{c}\text { inspection } \\
\text { records } \\
\text { number }\end{array}$ & $\begin{array}{c}\text { Recheck } \\
\text { number }\end{array}$ & $\begin{array}{c}\text { Accuracy } \\
/ \%\end{array}$ \\
\hline $\begin{array}{c}\text { Surface } \\
\text { crack }\end{array}$ & 17 & 15 & 17 & 100 \\
\hline $\begin{array}{c}\text { The paint } \\
\text { peeling off }\end{array}$ & 14 & 13 & 13 & 92.9 \\
\hline $\begin{array}{c}\text { Surface } \\
\text { corrosion }\end{array}$ & 19 & 17 & 18 & 94.7 \\
\hline $\begin{array}{c}\text { Repair } \\
\text { trace }\end{array}$ & 6 & 5 & 5 & 83.3 \\
\hline
\end{tabular}

As can be seen from the results in Table 1, the number of defects detected by this detection system is more than that recorded by wind farm inspection. This is because the traditional inspection method of wind farm 
is to use telescope observation or high-definition camera to take 360-degree photos of the blades, with limited resolution. After the on-site review of the number of defects detected in the system, the accuracy of cracks is nearly $100 \%$, which is related to the long length of cracks and high recognition degree. The accuracy of paint peeling and repairing marks is relatively low. The reasons are mainly caused by blade surface stain. At the same time, the number of samples trained by the system is relatively small. Once there are enough training samples, the detection accuracy will be improved. This test fully verified the high accuracy of the detection system in the detection of wind turbine blade defects.

\section{Conclusion}

In this paper, a wind turbine blade defect detection system was used to conduct blade defect detection test in a wind farm in Shandong province. Firstly, the camera array was used to collect images of two wind turbines blades with relatively more surface defects in the inspection process. HD01 image recognition software was used to detect, classify and locate defects in the collected images, and the results were statistically processed, which were compared with the traditional inspection method of wind farm blades. According to the experimental comparison results, the feasibility and applicability of the defect detection system to the nondestructive testing method of wind turbine blades are verified, the system proposed in this paper is based on the combination of imaging array and image processing server. The detection accuracy indicates that the detection system has an excellent detection effect, which verifies the advantages of the detection method such as high efficiency and high precision.

\section{Acknowledgement}

The auther gratefully acknowledge the contributions of the Science and technology project of China Huadian Corporation Ltd. (CHDKJ17-01-57)

\section{References}

1. Yang B,Sun D.Testing inspecting and monitoring technologies for wind turbine blades: A survey. Renewable \& Sustainable Energy Reviews, 22(8),515-526(2013).

2. ADAMS D,WHITE J, R UMSEY $M$, et al. Structural health monitoring of wind turbines: method and application to a HAWT. Wind Energy,14(4), 603-623 (2011).

3. Luping Li, Mangmang Li, Fenghua Jin, et al. The application of vibration detection technology in blade crack fault monitoring of wind turbine. Journal of Engineering for Thermal Energy and Power, 28 (2) , 208-222 (2013).

4. TANG J L, SOUA S, MAES C, et al. An experimental study of acoustic emission methodology for in service condition monitoring of wind turbine blades. Renewable Energy, 99, 170179 (2016).

5. REZAEIM M, BEHZAD M, MORADI $\mathrm{H}$, et al. Modal-based damage identification for the nonlinear model of modern wind turbine blade. Renewable Energy,94, 391-409 (2016).

6. Lee J K, Park J Y, Oh K Y, et al. Transformation algorithm of wind turbine blade moment signals for blade condition monitoring. Renewable Energy,79(1), 209-218(2014).

7. DOROSHTNASIR M, WORZEWSKI T, KRANKENHAGEN R, et al. On-site inspection of potential defects in wind turbine rotor blades with thermography. Wind Energy,19(8),1407-1422(2016). 